\title{
Predicament and Countermeasure of Regional Television Shopping Channel
}

\author{
Li-mei Zhou \\ Department of Politics and Law and Media, College of Information \& Business, Zhongyuan University of Technology, \\ Zhengzhou, Henan ,China
}

\begin{abstract}
From infomercial to TV shopping channel, TV shopping has been developing extremely rapidly. TV shopping channel in its coverage of different, can be divided into national and regional television shopping channel. Regional television shopping channel has to face competition from within the industry, but also to compete with online shopping and other new way to shop, face difficulties, developing new platforms and innovative program form, regional TV shopping channel can be healthy and sustainable development.
\end{abstract}

Keywords—regional, TV shopping, channel, predicament, countermeasure

\section{区域性电视购物频道的困境及对策}

\author{
周丽梅 \\ 中原工学院信息商务学院政法与传媒系, 郑州,河南,中国
}

\begin{abstract}
摘 要 电视购物从原先的购物节目到现如今的购物频道, 其发展异常迅速。电视购物频道以其覆盖范围为不同, 可分为全国性 电视购物频道及区域性电视购物频道。区域性电视购物频道既要面对来自行业内的竞争, 又要与网络购物等新兴的购物方式竞争, 正 视困境, 开拓新平台, 创新节目形态, 区域性电视购物频道才能健康、可持续发展。
\end{abstract}

关键词 区域性, 电视购物, 频道, 困境, 对策

随着我国经济的快速发展, 对外开放的程度加深, 国 人学习到了电视购物这一新型的无店铺销售方式, 被誉为 继零售、超市之后的第三次销售革命。电视购物在国内主 要以两种方式呈现: 一种是电视直销广告, 一种是电视购 物频道。早期电视直销广告夸大产品功效, 损害消费者的 利益，使得电视购物行业遭遇了信誉危机，后来以家庭购 物为理念的电视购物频道趁势崛起，与知名品牌合作，恢 复消费者的信心，打开了电视购物的新气象。国家对电视 购物行业也进行专项整治, 2014 年立案查处虚假违法电视 购物广告 691 件, 关停了 16 家非法开办的电视购物频道, 进一步规范了电视购物市场。如今电视购物以及新兴的网 络购物、手机购物等将零售渠道与媒介传播渠道相结合, 成为低成本、快捷购物的主要方式。当前, 区域性电视购 物频道既要面对全国性电视购物频道的扩张, 又面临新兴 购物方式的挑战, 发展遇到一定的困境, 只有不断开拓创 新, 才能实现可持续发展。

\section{1. 电视购物概述}

电视购物最早引起我国学者的关注是在 20 世纪 80 年 代, 理查德. 哈米尔顿博士, 美国直复营销学专家, 在首届 全国直复营销研讨会上向我国专家学者介始了电视营销的 实际应用, 也让我国专家第一次接触到了电视购物这一新 的营销方法。对于电视购物的定义, 学们者的观点相差不 大, 其中的区别最主要的在于是否包含了电视直销广告及 电视购物频道两种主要的类型。韩青、郑蔚在《电视服务 节目新论》中将电视购物节目专门列了一章进行分析, “电 视购物节目是依托电视媒体向观众详细介绍商品信息, 以 期立即产生购买行为的一种电视节目, 是电视购物这一销 售模式的重要组成部分”, 虽然没有对电视购物有一个明确 的定义, 但也提到了“电视购物从销售方来说也可以称作是 电视销售, 它是一种新型的营销模式。”[1]将电视购物归于 一种销售模式, 是许多学者共有的认识, “电视购物是以电 视台的专门购物频道为平台, 24 小时全天候不间断的以直 
播或录播的方式播出商品信息, 促进消费者认识、了解商 品信息后, 能够通过电视、电话、网络等途径订货, 由专 业物流公司在约定时间内配送商品上门, 通过第三方支付 或货到付款待方式回收货款的无店铺虚拟销售模式。”[2] 这一定义基本概括了电视购物的流程, 但将电视购物等同 于电视购物频道, 不免有些偏颇。“通过电视媒体发布商品 信息广告以及相应的热线电话号码, 由训练有素的话务员 进行“一对一”的悉心讲解及热情推荐最终达成销售, 然后通 过物流单位将商品送货上门、收回货款, 这种“无店铺销售” 模式就叫做电视购物。”麦奎尔在《大众传播理论》中提到 对电视购物的定义相对来说比较全面, 涵盖了电视直销有 电视购物频道两种形式, 但随着电视直销广告的日渐式微, 越来越多的人在谈到电视购物时会将其与电视购物频道相 联系。

\section{2. 区域性电视购物频道的发展概况}

电视购物频道是有线频道中的一种类型频道, 内容以 商业化性质内容播出, 目的在于销售商品以获取利润; 就 消费形式而言, 电视购物透过电视播放资讯广告, 消费者 透过电视获取商品资讯, 进而打电话订购, 是一种虚拟通 路的购物方式。[3]区域性电视购物频道, 是以电视购物频 道的覆盖范围为依据, 相较于全国性电视购物频道, 其覆 盖范围限于所在省或市的行政区划内的电视购物频道的统 称。到目前为止, 拥有全国性的数字电视购物频道的数量 只有 11 个, 剩下 30 多家都属于区域性电视购物频道。

区域性电视购物频道属于电视购物中的第二梯队, 但 其发展速度也不容小觑, 2008 年, 快乐购物, 上海东方购 物销售额达到 20 亿元左右, 河南的欢腾购物销售额也达到 了 10 亿元, 日均销售额更是在 80 万元左右。区域性购物 频道就覆盖范围而言较全国性购物频道弱, 但也有其自身 的优势。首先, 依托母体频道信誉, 树立消费者信心。区 域性电视购物频道大多由省级或市级电视频道转变而来, 原有频道受众对其有一定的认知度及认可度, 借助于省级 或市级媒体在本地区的信誉, 较易树立消费者对购物频道 所售产品的信心, 这对经历了电视直销广告信任危机的电 视购物产业来说显得尤为重要。其次, 立足特定区域, 深 耕本土市场。区域性电视购物频道利用原频道通路, 基本 可实现对本区域各地市、县、乡镇的全覆盖, 如果说全国 性购物频道在覆盖广度上要优于区域性购物频道, 那么在 深度上则不及区域性购物频道。区域性购物频道可深入分 析本区域消费者的消费特点及习惯, 对商品的挑选上更具 有针对性, 除一些家电、化妆品等大众的商品外, 对一些 在地消费类商品, 如教育类, 通讯服务类, 娱乐餐饮类等 亦可进行开发。

\section{3. 区域性电视购物频道面临的困境}

\section{1 覆盖范围受束缚}

台湾东森电视购物总经理宋湘岗直言: “电视购物赚的 是规模经济的钱! ”达不到一定的规模效应, 就会形成较大 的盈利压力。要达到一定的规模效应是以电视节目所覆盖 的人数为基础的, 所以覆盖范围从很大程度上决定了购物 频道的销售额。区域性电视购物频道从一开始就被束缚了 市场规模, 成为其自身销售额增长的最大瓶颈。随着湖南 快乐购、中视购物等全国性电视购物频道在全国扩张步伐 的加快, 区域内电视购物频道重叠覆盖率在不断上升, 竞 争异常激烈, 上海东方购也在江苏、浙江、湖北、甘肃、 广东、四川、山东等国内主要省区, 以及昆明、青岛等部 分重点城市落地, 截至 2014 年 6 月 30 日, 快乐购电视购 物节目覆盖 19 个省、自治区, 3 个直辖市, 全国共 11434 万户家庭可以同时通过快乐购物频道实现在线订购。全国 性电视购物频道正在逐步蚕食区域性电视购物频道的市 场, 使得后者面临较大的生存压力。如果不能有效地覆盖 较多的收视人群, 扩大市场规模, 摊薄单个商品的成本, 购物频道很难实现盈利, 覆盖范围的限定, 是区域性电视 购物频道面临的最大困境。

\section{2 同质化倾向严重}

2006 年是电视购物频道快速发展的一年, 中央媒体、 各省、市广电媒体纷纷开设购物频道, 频道数量急剧增加, 但大部分购物频道呈现出严重的同质化倾向。这种倾向表 现在两个方面, 一方面是购物频道之间的同质化。无论从 产品类别、节目形态, 运营方式上皆较为相似, 甚至连细 节上也很雷同, 屏幕上的字幕、位置、呈现方式几乎一模 一样, 使得各家购物频道的辨识度很低。各购物频道合作 的商品品牌同质化也较为严重。因电视购物因其固有的节 目形态供应商相对较少, 且同一个供应商经常横跨多个频 道, 一款多投, 进一步加剧了商品同质化现象。

另一方面每个时段呈现商品的方式过于雷同, 多数为 主持人加厂商代表或模特展示, 单一同质化的展示、推销 产品不易于消费者对商品的识别, 也容易造成审美疲劳。

\section{3 网络购物带来冲击}

根据中国互联网络信息中心 (CNNIC)发布的《第 35 次中国互联网络发展状况统计报告》称, 截至 2014 年 12 月, 我国网民规模达 6.49 亿, 手机网民规模更是达到了 5.57 亿, 较 2013 年增加了 5672 万人。[4]与网民规模增长相对 应的是中国网购物人数的急剧上升。截至 2014 年 12 月, 
中国网络购物的网民数量已经超过 3.61 亿人, 较 2013 年 底增加了 5953 万人, 增长率为 $19.7 \%$, 我国网民使用网络 购物的比例从 $48.9 \%$ 提升至 $55.7 \%$ 。更加直观的数据是, 天 猫商城首届“双十一”交易额为 0.5 亿元, 2013 年扩大到 350 亿元, 2014 年一举突破亿元大关, 网络购物势不可挡。谁 是网络的主体消费人群? 《CTR: 2011 年 Q3 受众媒介接 触习惯调查》中显示, 电视受众和视频受众就性别而言差 异不大, 偏女性。然而从年龄层来看, 两组受众呈现完全 相反的趋势, 电视受众更加老龄化, 45-64 岁的人群占了近 6 成。而视频受众的 67\% 是 15-34 岁的年轻人。在学历和收 入上电视受众和视频受众也有较大的差异, 电视受众以中 等学历的人群为主, 而视频受众近 7 成为高学历, 因此收 入在 3000 元以上的人群比例几乎是电视受众比例的 2 倍。 [5]由此可见, 网络受众多为年轻人, 学历较高, 对新事物 的接受能力强, 消费能力也普遍较高。

网络购物的强势崛起已经成为电视购物的主要竞争对 手。网络购物具有电视购物的所有优点, 低廉的价格, 快 捷的支付方式, 送货上门等, 一网在手, 足不出户买遍世 界。电视购物频道的扩张, 需要投入大量的资金, 与网络 只需要进行网站维护相比, 电视购物的成本在逐年增加。

\section{4. 区域性电视购物频道可持续发展对策}

在新媒体环境下, 区域性电视购物频道在现阶段不仅 要面对来自同行的竞争, 同样要面对网络购物、移动购物 等新型的购物方式的竞争, 但竞争不是绝对的态势, 竞争 中也蕴含着合作的机会。区域性电视购物频道要克服困境, 实现可持续发展离不开创新两个字, 包括渠道的创新, 节 目形态的创新。

\section{1 拓展渠道, 实现立体覆盖}

对区域性电视购物频道来说, 由于政策的限制, 其覆 盖范围仅限于所在省市的行政区划内, 对于一种规模经济 来说, 属于先天不足。但依据《广电总局关于电视购物频 道建设和管理的意见》, 广电总局鼓励合作发展, 优秀的购 物频道可以通过输出资金、团队、技术、管理等, 参与合 办其它购物频道, 管理规范、服务质量高、经营效益好的 购物频道, 可以逐步扩大覆盖范围。

\subsection{1 与异地电视台跨区域合作}

区域性电视购物频道全国扩张最理想的状态就是能让 整个购物频道的节目平移到其它区域进行播出, 这也是最 简单, 最方便的方法, 与异地电视台合作可基本实现这目 标。借鉴于电视直销广告购买播出时段的做法, 区域性电 视购物频道也可购买其它电视台的播出时段, 区域性电视
购物频道在本区域内有较健全的仓储物流配送体系, 如需 进行异地扩张, 本地配送的话物流成本过高, 当地的物流 公司合作, 减少人力成本, 但不可近因素过多, 也可选择 在异地建立配套的仓储, 物流体系, 但需投入的资金较大。 购买时段的方式与当地的电视台没有太多的利益珲扯, 属 于一种浅层的合作，购物频道自身具有较大的主控权。

共同运营购物频道是另一种深入合作方式, 区域性电 视购物频道与当地电视台合作, 由前者提供制作好的购物 节目, 后者编排播出, 利益分配可自行协商解决, 这是一 种共赢的合作方式, 目前已有一些成功的先例, 如深圳广 电集团与湖北电视台、济南电视台、郑州电视台等合作, 贵州电视台和甘肃合作等, 都取得了不错的成绩, 但也存 在当地购物频道成熟后自行建立购物频道的风险。

\subsection{2 整合新媒体新技术资源}

互联网的快速普及, 各类新媒体, 新技术的不断发展, 为区域性电视购物频道的渠道扩张带来的新的契机。《广电 总局印发关于电视购物频道建设和管理的意见的通知》中, 鼓励开办购物频道的播出机构及其控股的购物企业积极发 展网络广播电视、移动多媒体广播 ( CMMB)、手机电视、 IP 电视、互动点播等新媒体购物方式。据 CNNIC 研究显 示, 2014 年我国手机网络购物用户规模达到 2.36 亿, 增长 率为 $63.5 \%$, 是网络购物市场整体用户规模增长速度的 3.2 倍。电视购物行业中的快乐购、东方购物都相继开辟手机 客户端, 有数据显示, 2013 年开年 4 个月, 快乐购的移动 客户端及手机页面的销售额, 已接近整个快乐购网站的 $10 \%$, 而区域性电视购物频道如欢腾购物则还未开设手机 客户端。微博, 微信的飞速发展使得电商和社交平台的融 合显得尤为突出, 大多数电视购物频道都开通了微博或微 信官方帐号, 在这些社交平台上发布商品信息, 微信上还 可发布语音信息, 节目预告, 并可以打开相应的页面, 完 成商品的购买, 非常的便捷, 深受年轻人的喜爱。

城市中的移动电视频道, 户外电子屏也应成为区域性 电视购物频道渠道扩展的, 一般移动电视频道及户外电子 屏都放置在人流量较为密集的区域, 可作为特定区域覆盖 的补充。

\section{2 明确频道定位, 实现差异化竞争。}

面对日益激烈的市场竞争, 电视购物频道走专业化道 路将是未来主要的发展方向。央广电视购物频道开播时, 定位时尚女性, 认为时尚女性是有品质、高消费能力的人 群，正好与频道本身倡导的“理性消费”相契合。家有购物 侧重于家居用品类, 确立“家居用品, 天天特价”的定位, 则是通过主打某一类商品的销售来实现的。区域性电视购 
物频道的定位一方面要依托于母体频道的特色, 注重凝炼 频道母体的特色和文化理念, 适合于受众的审美倾向和接 受习惯。另一方面要关注本区域的经济发展状况, 重视区 域特色, 对本区域内受众的年龄构成、收入水平、消费习 惯等要进行整体分析, 作为商品篮选, 确定价格的主要依 据。

相较于全国性电视购物频道, 区域性电视购物频道的 产品分类可以更加细化, 突出区域特色, 如一些只可以在 当地消费的商品, 如教育类, 餐饮娱乐类, 如健身卡, 优 惠卡等。此外, 应多开通有第三方担保的网上快捷支付手 段, 适应现代年轻消费者的购物习惯。

\section{3 建设电视购物网站, 打造电子商务平台。}

根据美国、韩国等电视购物发展较为成熟的市场来看, 电视购物行业中, 电视购物网络所占的销售比正在逐年的 上升, 韩国 2013 年网络购物交易额首破 55 万亿韩元, 而 电视购物的比重从原先的 $16 \%$ 降至 $15 \%$ 。由此可见, 电视 购物网站正在成为电视购物行业除电视销售之外最重要的 渠道之一, 具有很大的发展潜力。电视购物频道的运营者 都已认识到网络购物的发展势头, 相继开设了电视购物网 站, 但对其重视不够, 很多只是作为电视购物频道的辅助 销售平台, 只设立了静态展示区, 建设的较为粗糙, 没有 长远的规划与设计。以河南电视台欢腾购物网站为例, 左 上角有非常醒目的“河南电视台欢腾购物频道”字样, 网站 首页导航栏较为简单大方, 导航条上专门设置了“欢腾官 网”一栏, 其实是河南天益电视购物有限公司的页面。网站 首页所展示产品有明确的归类, 开通了在线直播的视频窗 口, 可同步观看电视购物节目。右上方设有带有宣传性质 的活动公告栏, 作为购物网站给人一种格格不入的感觉。 整体来看, 欢腾购物网站可以基本满足消费者网上购物的 需求, 但还是带有门户网站的痕迹, 从网页设计上来说也 过于简单, 留白太多, 信息量小, 色彩也不够丰富, 点开 相关的产品后需后退才能返回首页继续汶览, 不方便, 相 比于其它购物网站, 对消费者的吸引有限。但有一点较为 突出的是借助于河南众多优秀的旅游资源, 欢腾购物网站 上专门开辟了欢腾旅游的栏目, 包含有省内、国内、境外
等旅游项目, 虽然还在建设当中, 但其产品意识应当肯定, 这也是差异化竞争的一种体现, 后期需与相关的旅游公司 进行深度的合作, 力争开辟出有区域特色, 能承载区域文 化的产品。

建设电视购物网站是在一定程度上也是区域性电视购 物频道突破电视销售渠道, 扩大市场规模的途径, 应把握 互联网快速发展的机遇, 转变思想, 开放思路, 将电视购 物网站打造成综合性的电子商务平台, 将其作为一个重要 的渠道来建设, 电视购物网站的设计、运营可借鉴优秀购 物网站, 如淘宝、京东、卓越等相对较为成熟的经验。

\section{5. 结语}

未来电视购物产业发展的内外部环境将会更加成熟, 国家对电视购物产业严格管理, 各项法律法规将会不断完 善, 引导电视购物产业走向有序化。电视购物行业的商业 模式发展成熟, 产业链分工明确。暴利型电视直销广告将 会慢慢淡出公众视野, 稳定利润型的家庭购物频道将成为 主流, 一些家庭购物优秀品牌将逐渐形成。遵循于电视购 物是规模经济的特点, 区域性电视购物频道将实现全国扩 张, 在媒体融合的趋势下, 将整合各新媒体新技术新产业, 打造综合性的电子商务平台。

\section{参考文献(References)}

[1] QingHan and WeiZheng, "New Theory of television program services," beijing: China Radio and Television Press,2005,pp.110

[2] Hong Zou and Fujuan Wang, "Under the credit crisis the way out TV shopping," Group text World, vol. 3,pp.90-101,2001.

[3] EHS Eastern Shopping Co, "TV shopping industry operators", beijin: Communication University of China Press,2011,pp.03-04

[4] Statistical Report on Internet Development of the 35th China InternetNetwork,

$\mathrm{DB} / \mathrm{OL}$ http://www.cnnic.cn/hlwfzyj/hlwxzbg/201502/P02015020355180 2054676.pdf

[5] CTR: Q3 audience media habits survey in 2011,DB/OL, http://www.199it.com/archives/22536.html. 